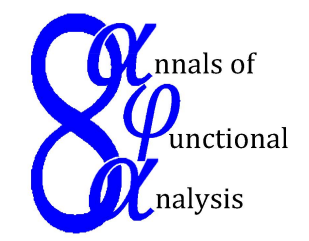

Ann. Funct. Anal. 6 (2015), no. 3, 60-72

http://doi.org/10.15352/afa/06-3-6

ISSN: 2008-8752 (electronic)

http://projecteuclid.org/afa

\title{
NECESSARY AND SUFFICIENT CONDITIONS FOR GENERALIZED HAUSDORFF OPERATORS AND COMMUTATORS
}

\author{
XIAOMEI WU \\ Communicated by V. Müller
}

\begin{abstract}
In this paper, we introduce a type of generalized Hausdorff operators and characterize the boundedness of these operators on Lebesgue spaces and central Morrey spaces. Moreover, we obtain the operator norms on these spaces. We also obtain sufficient and necessary conditions which ensure the boundedness of their commutators on Lebesgue spaces and central Morrey spaces with symbols in central BMO spaces. As applications, we give a new method to obtain sharp bounds for weighted Hardy operators and weighted Cesàro operators on Lebesgue spaces and central Morrey spaces.
\end{abstract}

\section{INTRODUCTION}

The study of Hausdorff operators has a long history in analysis. It can go back to the Hausdorff summability method which was introduced in 1917 in connecting with summability of number series. The modern (continuous) versions of Hausdorff operators were initiated with the work of Siskakas in complex analysis setting [16] and with the work of Georgakis [13] and Liflyand-Móricz in the Fourier transform setting [15]. Recently, an increasing attention has been acquired on the boundedness of Hausdorff operators and their commutators on various spaces and their sharp bounds. For an overview, we refer to [3], [5], [6], [7], [8], [10], [12], [14], [17] and references therein. The one dimensional Hausdorff operator is defined by

$$
h_{\Phi} f(x)=\int_{0}^{\infty} \frac{\Phi\left(\frac{x}{t}\right)}{t} f(t) d t, \quad x>0,
$$

Date: Received: Nov. 22, 2014; Accepted: Dec. 23, 2014.

2010 Mathematics Subject Classification. Primary 42B25; Secondary 42B35, 46E30.

Key words and phrases. Hausdorff operator, commutator, weighted Hardy operator, central Morrey space. 
where $\Phi(t)$ is a locally integrable function on $(0, \infty)$. By the generalized Minkowski inequality and the scaling property, one can easily show that $h_{\Phi}$ is bounded on $L^{p}\left(\mathbb{R}^{+}\right), 1 \leq p \leq \infty$, if

$$
\int_{0}^{\infty}|\Phi(t)| t^{-1+\frac{1}{p}} d t<\infty
$$

Hausdorff operator is connected to many well known operators in real and complex analysis. It is easy to verify that the Hardy operator

$$
h f(x)=\frac{1}{x} \int_{0}^{x} f(t) d t, x \neq 0,
$$

and its adjoint

$$
h^{*} f(x)=\int_{x}^{+\infty} \frac{f(t)}{t} d t
$$

are special cases of Hausdorff operator $h_{\Phi}$ if one replaces $\Phi$ with radial functions $\Phi_{1}(t)=t^{-1} \chi_{(1, \infty)}(t)$ and $\Phi_{2}(t)=\chi_{(0,1)}(t)$, respectively. Let $\phi:[0,1] \rightarrow[0, \infty)$ be a measurable function, if we take $\Phi_{3}(t)=\frac{\phi(1 / t)}{t} \chi_{(1, \infty)}(t)$, then the Hausdorff operator $h_{\Phi}$ will become the weighted Hardy operator defined by

$$
U_{\phi} f(x)=\int_{0}^{1} f(t x) \phi(t) d t, x \in \mathbb{R} .
$$

In 1984, Carton-Lebrun and Fosset in [4] first introduced the weighted Hardy operator in n-dimension. In [4], they obtained that $U_{\phi}$ is bounded from BMO into itself under certain conditions on $\phi$. In [18], Xiao got the BMO-norm of $U_{\phi}$, which optimizes the main results in [4]. In addition, Xiao found that $U_{\phi}$ is bounded on $L^{p}\left(\mathbb{R}^{n}\right), 1 \leq p \leq \infty$, if and only if

$$
\int_{0}^{1} t^{-\frac{n}{p}} \phi(t) d t<\infty
$$

and he showed that

$$
\left\|U_{\phi}\right\|_{L^{p}\left(\mathbb{R}^{n}\right) \rightarrow L^{p}\left(\mathbb{R}^{n}\right)}=\int_{0}^{1} t^{-\frac{n}{p}} \phi(t) d t .
$$

In 2012, Fu, Lu and Yuan ([11]), extended the results to the central Morrey spaces and considered the boundedness of their commutators on these spaces. By the analogues of singular and maximal operators associated with certain submanifolds of positive codimension in $\mathbb{R}^{n}$, in [9], Chuong and Hung extended $U_{\phi}$ to a generalized weighted Hardy-Cesàro operator $U_{\phi, s}$. Let $\phi:[0,1] \rightarrow[0, \infty), s:[0,1] \rightarrow \mathbb{R}$ be measurable functions. Then the generalized weighted Hardy-Cesáro operator $U_{\phi, s}$, associated to the parameter curves $s(x, t):=s(t) x$, is defined by

$$
U_{\phi, s}(f)(x)=\int_{0}^{1} f(s(t) x) \phi(t) d t
$$

for measurable complex-valued functions on $\mathbb{R}^{n}$. If $s(t)=t, U_{\phi, s}$ is reduced to $U_{\phi}$. Let $b$ be a locally integrable function on $\mathbb{R}^{n}$. The commutators of $b$ and operators $U_{\phi, s}$ is defined by

$$
U_{\phi, s}^{b}(f)(x)=b(x) U_{\phi, s}(f)(x)-U_{\phi, s}(b f)(x) .
$$


In [9], Chuong and Hung considered $U_{\phi, s}$ and their commutators in weighted $L^{p}$ spaces and BMO spaces.

Motivated by [9], in this paper, we consider a kind of generalized Hausdorff operators. Let $\Phi$ be a locally integrable function, $s$ be a measurable function on $\mathbb{R}^{+}$. Then the generalized Hausdorff operator, associated to the parameter curves $s(x, t):=s(t) x$, is defined by

$$
\mathcal{H}_{\Phi, s} f(x)=\int_{\mathbb{R}^{n}} \frac{\Phi(y)}{|y|^{n}} f(s(|y|) x) d y .
$$

In addition, its commutator is defined as

$\mathcal{H}_{\Phi, s}^{b} f(x)=b(x) \mathcal{H}_{\Phi, s} f(x)-\mathcal{H}_{\Phi, s}(b f)(x)=\int_{\mathbb{R}^{n}} \frac{\Phi(y)}{|y|^{n}} f(s(|y|) x)[b(x)-b(s(|y|) x)] d y$.

If $s(t)=\frac{1}{t}$, then $\mathcal{H}_{\Phi, s}$ is reduce to $H_{\Phi}$ defined by

$$
H_{\Phi} f(x)=\int_{\mathbb{R}^{n}} \frac{\Phi(y)}{|y|^{n}} f\left(\frac{x}{|y|}\right) d y,
$$

which is one of the extensions to high dimensional spaces, see [2]. There are other ways to extend $h_{\Phi}$, see [3], [5], [17] and the references therein for details. If $\Phi(t)=$ $t \phi(t) \chi_{(0,1)}(t)$, then $\mathcal{H}_{\Phi, s}$ will be equivalent to $U_{\phi, s}$. If $\Phi(t)=t^{n-1} \phi\left(\frac{1}{t}\right) \chi_{(1, \infty)}(t)$, then $\mathcal{H}_{\Phi, s}$ will be equivalent to $V_{\phi, s}$, which is defined as

$$
V_{\phi, s} f(x)=\int_{0}^{1} \frac{\phi(t)}{t^{n}} f\left(s\left(\frac{1}{t}\right) x\right) d t .
$$

Moreover, let $s(t)=t$, then it is the weighted Cesàro operator $V_{\phi}$, which is defined as follows

$$
V_{\phi} f(x)=\int_{0}^{1} \frac{\phi(t)}{t^{n}} f\left(\frac{x}{t}\right) d t .
$$

It is adjoint with the weighted Hardy operator $U_{\phi}$.

In what follows, we introduce some more definitions and notations. Let $B(0, r)$ denote the ball centered at 0 and with radius $r$. In [1], Alvararez, GuzmanPartida and Lakey introduced the central Morrey spaces.

Definition 1.1. Let $-\frac{1}{p} \leq \lambda<0$ and $1<p<\infty$.A function $f \in L_{l o c}^{p}\left(\mathbb{R}^{n}\right)$ is said to belong to the central Morrey spaces $\dot{B}^{p, \lambda}\left(\mathbb{R}^{n}\right)$ if and only if

$$
\|f\|_{\dot{B}^{p, \lambda}\left(\mathbb{R}^{n}\right)}=\sup _{r>0}\left(\frac{1}{|B(0, r)|^{1+\lambda p}} \int_{B(0, r)}|f(x)|^{p} d x\right)^{\frac{1}{p}}<\infty .
$$

When $\lambda=-\frac{1}{p}$, then $\dot{B}^{p, \lambda}\left(\mathbb{R}^{n}\right)=L^{p}\left(\mathbb{R}^{n}\right)$. One can easily check that $\dot{B}^{p, \lambda}\left(\mathbb{R}^{n}\right)$ reduces to $\{0\}$ when $\lambda<-1 / p$.

Definition 1.2. Let $1 \leq q<\infty$. A function $b \in L_{l o c}^{q}\left(\mathbb{R}^{n}\right)$ will be called in $C \dot{M} O^{q}\left(\mathbb{R}^{n}\right)$ if and only if

$$
\|b\|_{C \dot{M} O^{q}\left(\mathbb{R}^{n}\right)}=\sup _{r>0}\left(\frac{1}{|B(0, r)|} \int_{B(0, r)}\left|b(x)-b_{B}\right|^{q} d x\right)^{\frac{1}{q}}<\infty,
$$

where $b_{B}=\frac{1}{|B(0, r)|} \int_{B(0, r)} b(x) d x$. 
Further more, we can show that

$$
\begin{gathered}
B M O\left(\mathbb{R}^{n}\right) \subset C \dot{M} O^{q}\left(\mathbb{R}^{n}\right), 1 \leq q<\infty, \\
C \dot{M} O^{q_{1}}\left(\mathbb{R}^{n}\right) \subset C \dot{M} O^{q_{2}}\left(\mathbb{R}^{n}\right), 1 \leq q_{2}<q_{1}<\infty .
\end{gathered}
$$

When $q>1$, spaces $C \dot{M} O^{q}\left(\mathbb{R}^{n}\right)$ become Banach spaces after identifying the functions with differ by a constant almost everywhere. We refer for more details to $[1]$.

The main purpose of this article is to establish some boundedness properties of the generalized Hausdorff operators and their commutators in central Morrey spaces. The motivations of our results are the works in [9], [11] and [18]. More precisely, in Section 2, we devoted to the sharp estimates of $\mathcal{H}_{\Phi, s}$ on the Lebesgue spaces and the central Morrey spaces $\dot{B}^{p, \lambda}\left(\mathbb{R}^{n}\right)$. In Section 3, we establish the sufficient and necessary conditions on the function $\Phi$ which ensure the commutators $\mathcal{H}_{\Phi, s}^{b}$ with symbols in $C \dot{M} O^{p_{2}}\left(\mathbb{R}^{n}\right)$ are bounded from one central Morrey spaces $\dot{B}^{p_{1}, \lambda}\left(\mathbb{R}^{n}\right)$ to another central Morrey spaces $\dot{B}^{p, \lambda}\left(\mathbb{R}^{n}\right)$ and $p, p_{1}, p_{2}$ satisfy

the equality $\frac{1}{p}=\frac{1}{p_{1}}+\frac{1}{p_{2}}$. These results extend the theorems in [11] and [18]. As applications, we give a new method to obtain sharp bounds for weighted Hardy operators and weighted Cesàro operators on central Morrey spaces. Instead of standard methods in study of commutators of singular integrals by using sharp maximal functions to control commutators, the key of our methods is to get a pointwise estimate by using the boundedness of Hausdorff operators.

Throughout this paper, we write $A \preceq B$ to mean that $A \leq C B$ with some positive constant $\mathrm{C}$ independent of appropriate quantities.

\section{Sharp Boundedness of Generalized HausdorfF operators}

In this section, we discuss the sharp boundedness of generalized Hausdorff operators $\mathcal{H}_{\Phi, s}$ on Lebesgue spaces and central Morrey spaces. Our theorems are stated as follows.

Theorem 2.1. Let $1 \leq p<\infty,-\frac{1}{p} \leq \lambda<0$. Assume that $\Phi$ is a non-negative function. Let $s: \mathbb{R}^{+} \rightarrow \mathbb{R}$ be a measurable function such that $|s(t)|>0$ a.e. $t \in \mathbb{R}^{+}$. Denote

$$
\mathcal{A}=\int_{\mathbb{R}^{n}} \frac{\Phi(y)}{|y|^{n}}|s(|y|)|^{n \lambda} d y .
$$

Then

$$
\left\|\mathcal{H}_{\Phi, s} f\right\|_{\dot{B}^{p, \lambda}\left(\mathbb{R}^{n}\right)} \leq \mathcal{A}\|f\|_{\dot{B}^{p, \lambda}\left(\mathbb{R}^{n}\right)}
$$

if and only if $\mathcal{A}<\infty$. Moreover, we have

$$
\left\|\mathcal{H}_{\Phi, s}\right\|_{\dot{B}^{p, \lambda}\left(\mathbb{R}^{n}\right) \rightarrow \dot{B}^{p, \lambda}\left(\mathbb{R}^{n}\right)}=\mathcal{A} .
$$

Corollary 2.2. Let $1 \leq p<\infty,-\frac{1}{p} \leq \lambda<0$. Assume that $\phi$ is a non-negative function. Let $s: \mathbb{R}^{+} \rightarrow \mathbb{R}$ be a measurable function such that $|s(t)|>0$ a.e. $t \in \mathbb{R}^{+}$. Then

1)

$$
\left\|U_{\phi, s} f\right\|_{\dot{B}^{p, \lambda}\left(\mathbb{R}^{n}\right)} \leq \int_{0}^{1} \phi(t)|s(t)|^{n \lambda} d t\|f\|_{\dot{B}^{p, \lambda}\left(\mathbb{R}^{n}\right)}
$$


if and only if

$$
\int_{0}^{1} \phi(t)|s(t)|^{n \lambda} d t<\infty
$$

Moreover, we have

$$
\left\|U_{\phi, s}\right\|_{\dot{B}^{p, \lambda}\left(\mathbb{R}^{n}\right) \rightarrow \dot{B}^{p, \lambda}\left(\mathbb{R}^{n}\right)}=\int_{0}^{1} \phi(t)|s(t)|^{n \lambda} d t
$$

2)

$$
\left\|V_{\phi, s} f\right\|_{\dot{B}^{p, \lambda}\left(\mathbb{R}^{n}\right)} \leq \int_{0}^{1} \frac{\phi(t)}{t^{n}}\left|s\left(\frac{1}{t}\right)\right|^{n \lambda} d t\|f\|_{\dot{B}^{p, \lambda}\left(\mathbb{R}^{n}\right)}
$$

if and only if

$$
\int_{0}^{1} \frac{\phi(t)}{t^{n}}\left|s\left(\frac{1}{t}\right)\right|^{n \lambda} d t<\infty
$$

Moreover, we have

$$
\left\|V_{\phi, s}\right\|_{\dot{B}^{p, \lambda}\left(\mathbb{R}^{n}\right) \rightarrow \dot{B}^{p, \lambda}\left(\mathbb{R}^{n}\right)}=\int_{0}^{1} \frac{\phi(t)}{t^{n}}\left|s\left(\frac{1}{t}\right)\right|^{n \lambda} d t .
$$

Proof. 1) Taking $\Phi(y)=|y| \phi(|y|) \chi_{\{|y| \leq 1\}}(y)$, then by the polar decomposition, we obtain

$$
\mathcal{H}_{\Phi, s} f(x)=\omega_{n} \int_{0}^{1} \phi(t) f(s(t) x) d t=\omega_{n} U_{\phi, s} f(x)
$$

where $\omega_{n}$ denotes the Lebesgue measure of the unit sphere in $\mathbb{R}^{n}$.

2) Taking $\Phi(y)=\phi(1 /|y|)|y|^{n-1} \chi_{\{|y| \geq 1\}}(y)$, then by the polar decomposition, we obtain

$$
\mathcal{H}_{\Phi, s} f(x)=\omega_{n} \int_{0}^{1} \frac{\phi(t)}{t^{n}} f\left(s\left(\frac{1}{t}\right) x\right) d t=\omega_{n} V_{\phi, s} f(x) .
$$

Then the corollary is followed by Theorem 2.1 .

Remark 2.3. If we take $s(t)=\frac{1}{t}$ in Theorem 2.1, then by an easy calculation, we get

$$
\mathcal{A}=\int_{\mathbb{R}^{n}} \Phi(y)|y|^{-n-n \lambda} d y
$$

Therefore, $H_{\Phi}$ is bounded on $\dot{B}^{p, \lambda}\left(\mathbb{R}^{n}\right)$ if and only if

$$
\int_{\mathbb{R}^{n}} \Phi(y)|y|^{-n-n \lambda} d y<\infty
$$

where $-\frac{1}{p} \leq \lambda<0$. 
Proof of Theorem 2.1 By Minkowski's inequality and a change of variables, we have

$$
\begin{aligned}
\left\|\mathcal{H}_{\Phi, s} f\right\|_{L^{p}(B(0, r))} & =\left(\int_{B(0, r)}\left|\int_{\mathbb{R}^{n}} \frac{\Phi(y)}{|y|^{n}} f(s(|y|) x) d y\right|^{p} d x\right)^{\frac{1}{p}} \\
& \leq \int_{\mathbb{R}^{n}} \frac{|\Phi(y)|}{|y|^{n}}\left(\int_{B(0, r)}|f(s(|y|) x)|^{p} d x\right)^{\frac{1}{p}} d y \\
& =\int_{\mathbb{R}^{n}} \frac{|\Phi(y)|}{|y|^{n}}|s(|y|)|^{-\frac{n}{p}}\left(\int_{B(0,|s(|y|)| r)}|f(x)|^{p} d x\right)^{\frac{1}{p}} d y .
\end{aligned}
$$

We obtain

$$
\begin{aligned}
& \left\|\mathcal{H}_{\Phi, s} f\right\|_{\dot{B}^{p, \lambda}\left(\mathbb{R}^{n}\right)} \\
& \leq \sup _{r>0} \frac{1}{|B(0, r)|^{1 / p+\lambda}} \int_{\mathbb{R}^{n}} \frac{|\Phi(y)|}{|y|^{n}}|s(|y|)|^{-\frac{n}{p}}\left(\int_{B(0,|s(|y|)| r)}|f(x)|^{p} d x\right)^{\frac{1}{p}} d y \\
& =\int_{\mathbb{R}^{n}} \frac{|\Phi(y)|}{|y|^{n}}|s(|y|)|^{n \lambda} d y\|f\|_{\dot{B}^{p, \lambda}\left(\mathbb{R}^{n}\right)} .
\end{aligned}
$$

Thus, $\mathcal{H}_{\Phi, s}$ maps $\dot{B}^{p, \lambda}\left(\mathbb{R}^{n}\right)$ into itself and

$$
\left\|\mathcal{H}_{\Phi, s}\right\|_{\dot{B}^{p, \lambda}\left(\mathbb{R}^{n}\right) \rightarrow \dot{B}^{p, \lambda}\left(\mathbb{R}^{n}\right)} \leq \int_{\mathbb{R}^{n}} \frac{\Phi(y)}{|y|^{n}}|s(|y|)|^{n \lambda} d y .
$$

Conversely, to see the necessity, we prove it in two cases.

Case 1. If $-\frac{1}{p}<\lambda<0$, let $f_{0}(x)=|x|^{\lambda n}$. It is easy to check that $f_{0} \in \dot{B}^{p, \lambda}\left(\mathbb{R}^{n}\right)$ and $\left\|f_{0}\right\|_{\dot{B}^{p, \lambda}\left(\mathbb{R}^{n}\right)}=\omega_{n}^{-\lambda} n^{\frac{1}{p}+\lambda}(n \lambda p+n)^{-\frac{1}{p}}$. Then,

$$
\mathcal{H}_{\Phi, s} f_{0}(x)=|x|^{n \lambda} \int_{\mathbb{R}^{n}} \frac{\Phi(y)}{|y|^{n}}|s(|y|)|^{n \lambda} d y=f_{0}(x) \int_{\mathbb{R}^{n}} \frac{\Phi(y)}{|y|^{n}}|s(|y|)|^{n \lambda} d y .
$$

So,

$$
\left\|\mathcal{H}_{\Phi, s}\right\|_{\dot{B}^{p, \lambda}\left(\mathbb{R}^{n}\right) \rightarrow \dot{B}^{p, \lambda}\left(\mathbb{R}^{n}\right)} \geq \int_{\mathbb{R}^{n}} \frac{\Phi(y)}{|y|^{n}}|s(|y|)|^{n \lambda} d y
$$

It follows that $\mathcal{A}<\infty$. Therefore, combining (2.1) and (2.2), we get

$$
\left\|\mathcal{H}_{\Phi, s}\right\|_{\dot{B}^{p, \lambda}\left(\mathbb{R}^{n}\right) \rightarrow \dot{B}^{p, \lambda}\left(\mathbb{R}^{n}\right)}=\int_{\mathbb{R}^{n}} \frac{\Phi(y)}{|y|^{n}}|s(|y|)|^{n \lambda} d y .
$$

Case 2. If $\lambda=-\frac{1}{p}$, then for any $\epsilon>0$, we take $f_{1}(x)=|x|^{-\frac{n}{p}-\epsilon} \chi_{\{|x| \geq 1\}}(x)$. By an elementary calculation, we have $\left\|f_{1}\right\|_{L^{p}\left(\mathbb{R}^{n}\right)}=\omega_{n}^{\frac{1}{p}}(\epsilon p)^{-\frac{1}{p}}$. Denote $S(x, y)=$ $\{y|| s(|y|) x \mid \geq 1\}$, then

$$
\mathcal{H}_{\Phi, s} f_{1}(x)=|x|^{-n / p-\epsilon} \int_{S(x, y)} \frac{\Phi(y)}{|y|^{n}}|s(|y|)|^{-n / p-\epsilon} d y .
$$


For any $\epsilon>0$, if $|x|>\frac{1}{\epsilon}$, then $S\left(\frac{1}{\epsilon}, y\right) \subset S(x, y)$. Thus, we have

$$
\begin{aligned}
& \left\|\mathcal{H}_{\Phi, s} f_{1}\right\|_{L^{p}\left(\mathbb{R}^{n}\right)}=\left(\left.\left.\int_{\mathbb{R}^{n}}\left|\int_{S(x, y)} \frac{\Phi(y)}{|y|^{n}}\right| s(|y|)\right|^{-n / p-\epsilon} d y\right|^{p}|x|^{-n-p \epsilon} d x\right)^{\frac{1}{p}} \\
& \geq\left(\left.\left.\int_{|x|>\frac{1}{\epsilon}}\left|\int_{S\left(\frac{1}{\epsilon}, y\right)} \frac{\Phi(y)}{|y|^{n}}\right| s(|y|)\right|^{-n / p-\epsilon} d y\right|^{p}|x|^{-n-p \epsilon} d x\right)^{\frac{1}{p}} \\
& =\omega_{n}^{\frac{1}{p}}(\epsilon p)^{-\frac{1}{p}} \epsilon^{\epsilon} \int_{S\left(\frac{1}{\epsilon}, y\right)} \frac{\Phi(y)}{|y|^{n}}|s(|y|)|^{-n / p-\epsilon} d y \\
& =\epsilon^{\epsilon}\left\|f_{1}\right\|_{L^{p}\left(\mathbb{R}^{n}\right)} \int_{S\left(\frac{1}{\epsilon}, y\right)} \frac{\Phi(y)}{|y|^{n}}|s(|y|)|^{-n / p-\epsilon} d y .
\end{aligned}
$$

Letting $\epsilon \rightarrow 0$ (noticing $\epsilon^{\epsilon} \rightarrow 1$ ), then

$$
\left\|\mathcal{H}_{\Phi, s}\right\|_{L^{p}\left(\mathbb{R}^{n}\right) \rightarrow L^{p}\left(\mathbb{R}^{n}\right)} \geq \int_{\mathbb{R}^{n}} \frac{\Phi(y)}{|y|^{n}}|s(|y|)|^{-n / p} d y .
$$

So, by (2.1) and (2.3), we obtain

$$
\left\|\mathcal{H}_{\Phi, s}\right\|_{L^{p}\left(\mathbb{R}^{n}\right) \rightarrow L^{p}\left(\mathbb{R}^{n}\right)}=\int_{\mathbb{R}^{n}} \frac{\Phi(y)}{|y|^{n}}|s(|y|)|^{-n / p} d y .
$$

This completes the proof of Theorem 2.1.

\section{Commutators of generalized Hausdorff operators}

Recently, in [9], the authors obtained a necessary and sufficient condition on the function $\phi$, which ensures the boundedness of the generalized Hardy-Cesàro operators and its commutators on weighted $L^{p}$ spaces and BMO spaces. The purpose of this section is to establish a necessary and sufficient condition on the function $\Phi$, which ensures the boundedness of the commutators with symbols in $C \dot{M} O^{p}\left(\mathbb{R}^{n}\right)$ on central Morrey spaces. Our results extend the main theorems in [11].

The main theorems in this section are formulated as follows.

Theorem 3.1. Let $1 \leq p<p_{1}<\infty, \frac{1}{p}=\frac{1}{p_{1}}+\frac{1}{p_{2}},-\frac{1}{p} \leq \lambda<0$, let $b(x) \in$ $C \dot{M} O^{p_{2}}\left(\mathbb{R}^{n}\right)$ and $s: \mathbb{R}^{+} \rightarrow \mathbb{R}$ be a measurable function such that $|s(t)|>0$. Denote

$$
\mathcal{B}=\int_{\mathbb{R}^{n}} \frac{|\Phi(y)|}{|y|^{n}}|s(|y|)|^{n \lambda} \log \max \left\{2|s(|y|)|, \frac{2}{|s(|y|)|}\right\} d y .
$$

If $\mathcal{B}<\infty$, then $\mathcal{H}_{\Phi, s}^{b}$ is bounded from $\dot{B}^{p_{1}, \lambda}\left(\mathbb{R}^{n}\right)$ to $\dot{B}^{p, \lambda}\left(\mathbb{R}^{n}\right)$. That is

$$
\left\|\mathcal{H}_{\Phi, s}^{b} f\right\|_{\dot{B}^{p, \lambda}\left(\mathbb{R}^{n}\right)} \preceq \mathcal{B}\|b\|_{C \dot{M} O^{p_{2}}\left(\mathbb{R}^{n}\right)}\|f\|_{\dot{B}^{p_{1}, \lambda}\left(\mathbb{R}^{n}\right)} .
$$

Define new types of commutators generated by Hausdorff operators as follows.

$$
\mathcal{H}_{\Phi, 1}^{b} f(x)=\int_{|y| \leq 1} \frac{\Phi(y)}{|y|^{n}} f(s(|y|) x)[b(x)-b(s(|y|) x)] d y
$$




$$
\mathcal{H}_{\Phi, 2}^{b} f(x)=\int_{|y| \geq 1} \frac{\Phi(y)}{|y|^{n}} f(s(|y|) x)[b(x)-b(s(|y|) x)] d y .
$$

Then, we can obtain the following sufficient and necessary conditions on $\Phi$ which ensure the boundedness of the commutators on $\dot{B}^{p, \lambda}\left(\mathbb{R}^{n}\right)$.

Theorem 3.2. Let $1 \leq p<p_{1}<\infty, \frac{1}{p}=\frac{1}{p_{1}}+\frac{1}{p_{2}},-\frac{1}{p} \leq \lambda<0, b \in C \dot{M} O^{p_{2}}\left(\mathbb{R}^{n}\right)$. Assume that $\Phi$ is a non-negative integrable function.

1) Let $s: \mathbb{R}^{+} \rightarrow \mathbb{R}$ be a measurable function such that $t^{\beta} \leq|s(t)| \leq t^{\gamma}$ a.e. $t \in \mathbb{R}^{+}$for some constants $\beta, \gamma>0$. If we denote

$$
\mathcal{C}_{1}=\int_{|y| \leq 1} \frac{\Phi(y)}{|y|^{n}}|s(|y|)|^{n \lambda} \log 2 /|s(|y|)| d y,
$$

then $\mathcal{H}_{\Phi, 1}^{b}$ is bounded from $\dot{B}^{p_{1}, \lambda}\left(\mathbb{R}^{n}\right)$ to $\dot{B}^{p, \lambda}\left(\mathbb{R}^{n}\right)$ if and only if $\mathcal{C}_{1}<\infty$.

2) Let $s: \mathbb{R}^{+} \rightarrow \mathbb{R}$ be a measurable function such that $|s(t)| \geq t^{\gamma}$ a.e. $t \in \mathbb{R}^{+}$ for some constant $\gamma>0$. If we denote

$$
\mathcal{C}_{2}=\int_{|y| \geq 1} \frac{\Phi(y)}{|y|^{n}}|s(|y|)|^{n \lambda} \log 2|s(|y|)| d y,
$$

then $\mathcal{H}_{\Phi, 2}^{b}$ is bounded from $\dot{B}^{p_{1}, \lambda}\left(\mathbb{R}^{n}\right)$ to $\dot{B}^{p, \lambda}\left(\mathbb{R}^{n}\right)$ if and only if $\mathcal{C}_{2}<\infty$.

Corollary 3.3. Let $1 \leq p<p_{1}<\infty, \frac{1}{p}=\frac{1}{p_{1}}+\frac{1}{p_{2}},-\frac{1}{p} \leq \lambda<0, b \in C \dot{M} O^{p_{2}}\left(\mathbb{R}^{n}\right)$.

1) Let $s: \mathbb{R}^{+} \rightarrow \mathbb{R}$ be a measurable function such that $t^{\beta} \leq|s(t)| \leq t^{\gamma}$ a.e. $t \in \mathbb{R}^{+}$for some constants $\beta, \gamma>0$. Then $U_{\phi, s}^{b}$ is bounded from $\dot{B}^{p_{1}, \lambda}\left(\mathbb{R}^{n}\right)$ to $\dot{B}^{p, \lambda}\left(\mathbb{R}^{n}\right)$ if and only if

$$
\int_{0}^{1} \phi(t)|s(t)|^{n \lambda} \log \frac{2}{|s(t)|} d t<\infty
$$

2) Let $s: \mathbb{R}^{+} \rightarrow \mathbb{R}$ be a measurable function such that $|s(t)| \geq t^{\gamma}$ a.e. $t \in \mathbb{R}^{+}$ for some constant $\gamma>0$. Then $V_{\phi, s}^{b}$ is bounded from $\dot{B}^{p_{1}, \lambda}\left(\mathbb{R}^{n}\right)$ to $\dot{B}^{p, \lambda}\left(\mathbb{R}^{n}\right)$ if and only if

$$
\int_{0}^{1} \frac{\phi(t)}{t^{n}}\left|s\left(\frac{1}{t}\right)\right|^{n \lambda} \log 2\left|s\left(\frac{1}{t}\right)\right| d t<\infty
$$

The proof of this corollary is as similar as the proof of Corollary 2.2, we omit the details here.

Remark 3.4. If we assume $s(t)=t$, then we get the following results for the commutators of weighted Hardy operator $U_{\phi}^{b} f(x):=b(x) U_{\phi}(f)(x)-U_{\phi}(b f)(x)$. Namely, if $p, p_{1}, p_{2}$ and $\lambda$ satisfy the conditions as in Theorem 3.2, then for any $b \in C \dot{M} O^{p_{2}}\left(\mathbb{R}^{n}\right), U_{\phi}^{b}$ is bounded from $\dot{B}^{p_{1}, \lambda}\left(\mathbb{R}^{n}\right)$ to $\dot{B}^{p, \lambda}\left(\mathbb{R}^{n}\right)$ if and only if

$$
\int_{0}^{1} \phi(t) t^{n \lambda} \log \frac{2}{t} d t<\infty
$$

Under the same conditions on $p, p_{1}, p_{2}$ and $\lambda$, the result for the commutators of weighted Cesàro operator $V_{\phi}^{b} f(x):=b(x) V_{\phi}(f)(x)-V_{\phi}(b f)(x)$ is stated as follows. 
$V_{\phi}^{b}$ is bounded from $\dot{B}^{p_{1}, \lambda}\left(\mathbb{R}^{n}\right)$ to $\dot{B}^{p, \lambda}\left(\mathbb{R}^{n}\right)$ if and only if

$$
\int_{0}^{1} \frac{\phi(t)}{t^{n+n \lambda}} \log \frac{2}{t} d t<\infty .
$$

This is one of the main results in [11].

Now we are in a position to prove the theorems.

Proof of Theorem 3.1 Following, we will show that $\mathcal{H}_{\Phi, s}^{b}$ is a bounded operator from $\dot{B}^{p_{1}, \lambda}\left(\mathbb{R}^{n}\right)$ to $\dot{B}^{p, \lambda}\left(\mathbb{R}^{n}\right)$. For any ball $B(0, r)$ in $\mathbb{R}^{n}$, one has

$$
\begin{aligned}
& \left\|\mathcal{H}_{\Phi, s}^{b} f\right\|_{\dot{B}^{p, \lambda}\left(\mathbb{R}^{n}\right)} \\
& \leq \sup _{r>0}|B(0, r)|^{-\frac{1}{p}-\lambda}\left(\int_{B(0, r)}\left|\int_{\mathbb{R}^{n}} \frac{\Phi(y)}{|y|^{n}} f(s(|y|) x)\left[b(x)-b_{B}\right] d y\right|^{p} d x\right)^{\frac{1}{p}} \\
& +\sup _{r>0}|B(0, r)|^{-\frac{1}{p}-\lambda}\left(\int_{B(0, r)}\left|\int_{\mathbb{R}^{n}} \frac{\Phi(y)}{|y|^{n}} f(s(|y|) x)\left[b_{B}-b_{|s(|y|)| B}\right] d y\right|^{p} d x\right)^{\frac{1}{p}} \\
& +\sup _{r>0}|B(0, r)|^{-\frac{1}{p}-\lambda}\left(\int_{B(0, r)}\left|\int_{\mathbb{R}^{n}} \frac{\Phi(y)}{|y|^{n}} f(s(|y|) x)\left[b_{|s(|y|)| B}-b(s(|y|) x)\right] d y\right|^{p} d x\right)^{\frac{1}{p}} \\
& :=I+I I+I I I,
\end{aligned}
$$

where $b_{B}=\frac{1}{|B(0, r)|} \int_{B(0, r)} f(x) d x$. First, let us estimate I. Since $\frac{1}{p}=\frac{1}{p_{1}}+\frac{1}{p_{2}}$, by Hölder's inequality and Theorem 2.1, we have

$$
\begin{aligned}
I & =\sup _{r>0}|B(0, r)|^{-\frac{1}{p}-\lambda}\left(\int_{B(0, r)}\left|\mathcal{H}_{\Phi, s} f(x)\right|^{p}\left|b(x)-b_{B}\right|^{p} d x\right)^{\frac{1}{p}} \\
& \leq \sup _{r>0}|B(0, r)|^{-\frac{1}{p}-\lambda}\left(\int_{B(0, r)}\left|\mathcal{H}_{\Phi, s} f(x)\right|^{p_{1}} d x\right)^{\frac{1}{p_{1}}}\left(\int_{B(0, r)}\left|b(x)-b_{B}\right|^{p_{2}} d x\right)^{\frac{1}{p_{2}}} \\
& \leq \int_{\mathbb{R}^{n}} \frac{|\Phi(y)|}{|y|^{n}}|s(|y|)|^{n \lambda} d y\|b\|_{C \dot{M} O^{p_{2}}\left(\mathbb{R}^{n}\right)}\|f\|_{\dot{B}^{p_{1}, \lambda}\left(\mathbb{R}^{n}\right)} .
\end{aligned}
$$

For II, we divide it into two parts as follows. Using Minkowski's inequality, one has

II

$$
\begin{aligned}
& \leq \sup _{r>0}|B(0, r)|^{-\frac{1}{p}-\lambda} \int_{\mathbb{R}^{n}} \frac{\Phi(y)}{|y|^{n}}\left(\int_{B(0, r)}\left|f(s(|y|) x)\left[b_{B}-b_{|s(|y|)| B}\right]\right|^{p} d x\right)^{\frac{1}{p}} d y \\
& \leq \sup _{r>0}|B(0, r)|^{-\frac{1}{p}-\lambda} \int_{|s(|y|)| \leq 1} \frac{|\Phi(y)|}{|y|^{n}}\left(\int_{B(0, r)}\left|f(s(|y|) x)\left[b_{B}-b_{|s(|y|)| B}\right]\right|^{p} d x\right)^{\frac{1}{p}} d y \\
& +\sup _{r>0}|B(0, r)|^{-\frac{1}{p}-\lambda} \int_{|s(|y|)| \geq 1} \frac{|\Phi(y)|}{|y|^{n}}\left(\int_{B(0, r)}\left|f(s(|y|) x)\left[b_{B}-b_{|s(|y|)| B}\right]\right|^{p} d x\right)^{\frac{1}{p}} d y \\
& :=I I_{1}+I I_{2} .
\end{aligned}
$$

First, we estimate $I I_{1}$. For any $0<|s(|y|)| \leq 1$, there exists a $k \in \mathbb{Z}^{+}$, such that $2^{k-1} \leq|s(|y|)|^{-1}<2^{k}$. Then due to Hölder's inequality and changing of 
variables, we get

$I I_{1} \leq\|f\|_{\dot{B}^{p_{1}, \lambda}\left(\mathbb{R}^{n}\right)} \int_{|s(|y|)| \leq 1} \frac{|\Phi(y)|}{|y|^{n}}|s(|y|)|^{n \lambda}\left(\sum_{i=1}^{k}\left|b_{2^{-i} B}-b_{2^{-i+1} B}\right|+\left|b_{2^{-k} B}-b_{|s(|y|)| B}\right|\right) d y$.

Using Hölder's inequality, for any $i \in \mathbb{Z}, q \geq 1$, then in the inside integral, we obtain

$$
\begin{aligned}
\left|b_{2^{i} B}-b_{2^{i-1} B}\right| & =\frac{1}{\left|2^{i-1} B\right|}\left|\int_{2^{i-1} B}\left(b(x)-b_{2^{i} B}\right) d x\right| \preceq \frac{1}{\left|2^{i} B\right|}\left|\int_{2^{i} B}\left(b(x)-b_{2^{i} B}\right) d x\right| \\
& \leq\left(\frac{1}{\left|2^{i} B\right|} \int_{2^{i} B}\left|b(x)-b_{2^{i} B}\right|^{q} d x\right)^{\frac{1}{q}} \leq\|b\|_{C \dot{M} O^{q}\left(\mathbb{R}^{n}\right)} .
\end{aligned}
$$

It follows that

$$
\begin{aligned}
& I I_{1} \\
& \preceq \sum_{k=0}^{\infty} \int_{2^{-k-1} \leq|s(|y|)| \leq 2^{-k}} \frac{|\Phi(y)|}{|y|^{n}}|s(|y|)|^{n \lambda}(k+1) d y\|b\|_{C \dot{M} O^{p_{2}}\left(\mathbb{R}^{n}\right)}\|f\|_{\dot{B}^{p_{1}, \lambda}\left(\mathbb{R}^{n}\right)} \\
& \preceq \sum_{k=0}^{\infty} \int_{2^{-k-1} \leq|s(|y|)| \leq 2^{-k}} \frac{|\Phi(y)|}{|y|^{n}}|s(|y|)|^{n \lambda}\left(\log 2^{k}+1\right) d y\|b\|_{C \dot{M} O^{p_{2}}\left(\mathbb{R}^{n}\right)}\|f\|_{\dot{B}^{p_{1}, \lambda}\left(\mathbb{R}^{n}\right)} \\
& \leq \sum_{k=o}^{\infty} \int_{2^{-k-1} \leq|s(|y|)| \leq 2^{-k}} \frac{|\Phi(y)|}{|y|^{n}}|s(|y|)|^{n \lambda}\left(\log \frac{1}{|s(|y|)|}+1\right) \\
& d y\|b\|_{C \dot{M} O^{p_{2}}\left(\mathbb{R}^{n}\right)}\|f\|_{\dot{B}^{p_{1}, \lambda}\left(\mathbb{R}^{n}\right)} \\
& \leq \int_{|s(|y|)| \leq 1} \frac{|\Phi(y)|}{|y|^{n}}|s(|y|)|^{n \lambda}\left(\log \frac{1}{|s(|y|)|}+1\right) d y\|b\|_{C \dot{M} O^{p_{2}}\left(\mathbb{R}^{n}\right)}\|f\|_{\dot{B}^{p_{1}, \lambda}\left(\mathbb{R}^{n}\right)} \\
& \leq \int_{|s(|y|)| \leq 1} \frac{|\Phi(y)|}{|y|^{n}}|s(|y|)|^{n \lambda}\left(\log \frac{2}{|s(|y|)|}\right) d y\|b\|_{C \dot{M} O^{p_{2}}\left(\mathbb{R}^{n}\right)}\|f\|_{\dot{B}^{p_{1}, \lambda}\left(\mathbb{R}^{n}\right)} .
\end{aligned}
$$

Using an argument similar to the proof of $I I_{1}$, we have

$$
I I_{2} \leq \int_{|s(|y|)| \geq 1} \frac{|\Phi(y)|}{|y|^{n}}|s(|y|)|^{n \lambda}(\log 2|s(|y|)|) d y\|b\|_{C \dot{M} O^{p_{2}}\left(\mathbb{R}^{n}\right)}\|f\|_{\dot{B}^{p_{1}, \lambda}\left(\mathbb{R}^{n}\right)} .
$$

Finally, we estimate III. By Minkowski's inequality and Hölder's inequality, we have

$$
I I I \leq \sup _{r>0}|B(0, r)|^{-\frac{1}{p}-\lambda} \int_{\mathbb{R}^{n}} \frac{|\Phi(y)|}{|y|^{n}}\left(\int_{B(0, r)}\left|f(s(|y|) x)\left[b_{|s(|y|)| B}-b(s(|y|) x)\right]\right|^{p} d x\right)^{\frac{1}{p}} d y
$$




$$
\begin{aligned}
& \leq \sup _{r>0}|B(0, r)|^{-\frac{1}{p}-\lambda} \int_{\mathbb{R}^{n}} \frac{|\Phi(y)|}{|y|^{n}}\left(\int_{B(0, r)}|f(s(|y|) x)|^{p_{1}} d x\right)^{\frac{1}{p_{1}}} \\
& \cdot\left(\int_{B(0, r)}\left|b_{|s(|y|)| B}-b(s(|y|) x)\right|^{p_{2}} d x\right)^{\frac{1}{p_{2}}} d y \\
& \leq \sup _{r>0}|B(0, r)|^{-\frac{1}{p}-\lambda} \int_{\mathbb{R}^{n}} \frac{|\Phi(y)|}{|y|^{n}}|s(|y|)|^{-\frac{n}{p}}\left(\int_{B(0, r|s(|y|)|)}|f(x)|^{p_{1}} d x\right)^{\frac{1}{p_{1}}} \\
& \cdot\left(\int_{B(0, r|s(|y|)|)}\left|b_{|s(|y|)| B}-b(x)\right|^{p_{2}} d x\right)^{\frac{1}{p_{2}}} d y \\
& \leq \int_{\mathbb{R}^{n}} \frac{|\Phi(y)|}{|y|^{n}}|s(|y|)|^{n \lambda} d y\|b\|_{C \dot{M} O^{p_{2}}\left(\mathbb{R}^{n}\right)}\|f\|_{\dot{B}^{p_{1}, \lambda}\left(\mathbb{R}^{n}\right)} .
\end{aligned}
$$

Combining I, II and III, we have proved Theorem 3.1.

Proof of Theorem 3.2 1) The sufficient part is similar to the proof of Theorem 3.1. We only need to give the proof of the necessary part.

Case 1. If $-\frac{1}{p}<\lambda<0$, we take $f_{0}(x)=|x|^{\lambda n}$. A routine computation by the polar transformation shows that $\left\|f_{0}\right\|_{\dot{B}^{p_{1}, \lambda}\left(\mathbb{R}^{n}\right)}=\omega_{n}^{-\lambda} n^{\frac{1}{p_{1}}+\lambda}\left(\lambda p_{1} n+n\right)^{-\frac{1}{p_{1}}}<\infty$. So $f_{0} \in \dot{B}^{p_{1}, \lambda}\left(\mathbb{R}^{n}\right)$. Let $b_{0}(x)=\log |x|, x \in \mathbb{R}^{n}$, then $b_{0} \in B M O\left(\mathbb{R}^{n}\right) \subset C \dot{M} O^{p_{2}}\left(\mathbb{R}^{n}\right)$ and

$$
\mathcal{H}_{\Phi, 1}^{b_{0}} f_{0}(x)=|x|^{n \lambda} \int_{|y| \leq 1} \frac{\Phi(y)}{|y|^{n}}|s(|y|)|^{n \lambda} \log \frac{1}{|s(|y|)|} d y .
$$

Since $0<|s(|y|)| \leq|y|^{\gamma} \leq 1$, we have

$$
\begin{aligned}
\left\|\mathcal{H}_{\Phi, 1}^{b_{0}} f_{0}\right\|_{\dot{B}^{p, \lambda}\left(\mathbb{R}^{n}\right)} & =\int_{|y| \leq 1} \frac{\Phi(y)}{|y|^{n}}|s(|y|)|^{n \lambda} \log \frac{1}{|s(|y|)|} d y\left\|f_{0}\right\|_{\dot{B}^{p, \lambda}\left(\mathbb{R}^{n}\right)} \\
& =\omega_{n}^{-\lambda} n^{\frac{1}{p}+\lambda}(\lambda p n+n)^{-\frac{1}{p}} \int_{|y| \leq 1} \frac{\Phi(y)}{|y|^{n}}|s(|y|)|^{n \lambda} \log \frac{1}{|s(|y|)|} d y .
\end{aligned}
$$

So,

$$
\left\|\mathcal{H}_{\Phi, 1}^{b_{0}}\right\|_{\dot{B}^{p_{1}, \lambda}\left(\mathbb{R}^{n}\right) \rightarrow \dot{B}^{p, \lambda}\left(\mathbb{R}^{n}\right)} \succeq \int_{|y| \leq 1} \frac{\Phi(y)}{|y|^{n}}|s(|y|)|^{n \lambda} \log \frac{1}{|s(|y|)|} d y .
$$

Therefore, we obtain

$$
\int_{|y| \leq 1} \frac{\Phi(y)}{|y|^{n}}|s(|y|)|^{n \lambda} \log \frac{1}{|s(|y|)|} d y<\infty .
$$

For $|s(|y|)| \leq|y|^{\gamma}$, it follows that

$$
\int_{|y| \leq \frac{1}{2}} \frac{\Phi(y)}{|y|^{n}}|s(|y|)|^{n \lambda} d y \preceq \int_{|y| \leq \frac{1}{2}} \frac{\Phi(y)}{|y|^{n}}|s(|y|)|^{n \lambda} \log \frac{1}{|s(|y|)|} d y<\infty .
$$


On the other hand, for $\Phi(y)$ is an integral function and $|s(|y|)| \geq|y|^{\beta}(\beta>0)$ and $\lambda<0$, we know that

$$
\int_{\frac{1}{2} \leq|y| \leq 1} \frac{\Phi(y)}{|y|^{n}}|s(|y|)|^{n \lambda} d y<\infty
$$

According to (3.2) and (3.3), we have

$$
\int_{|y| \leq 1} \frac{\Phi(y)}{|y|^{n}}|s(|y|)|^{n \lambda} d y<\infty
$$

Noticing that

$$
\mathcal{C}_{1}=\log 2 \int_{|y| \leq 1} \frac{\Phi(y)}{|y|^{n}}|s(|y|)|^{n \lambda} d y+\int_{|y| \leq 1} \frac{\Phi(y)}{|y|^{n}}|s(|y|)|^{n \lambda} \log \frac{1}{|s(|y|)|} d y .
$$

Then, combining (3.1) and (3.4), we have $\mathcal{C}_{1}<\infty$.

Case 2. If $\lambda=-\frac{1}{p}$, then for any $0<\epsilon<\frac{1}{2}$, let $f_{1}(x)=|x|^{-\frac{n}{p}-\epsilon} \chi_{\{|x| \geq 1\}}(x)$. By a routine calculation, we have $\left\|f_{1}\right\|_{L^{p}\left(\mathbb{R}^{n}\right)}=\omega_{n}^{\frac{1}{p}}(\epsilon p)^{-\frac{1}{p}}$. Let $b_{0}(x)=\log |x|, x \in \mathbb{R}^{n}$, then $b_{0} \in C \dot{M} O^{p_{2}}\left(\mathbb{R}^{n}\right)$ and we have

$$
\mathcal{H}_{\Phi}^{b_{0}} f_{1}(x)=|x|^{-n / p-\epsilon} \int_{\{y|| y \mid \leq 1\} \cap S(x, y)} \frac{\Phi(y)}{|y|^{n}}|s(|y|)|^{-n / p-\epsilon} \log \frac{1}{|s(|y|)|} d y,
$$

where $S(x, y)=\{y|| s(|y|) x \mid \geq 1\}$. Thus, for any $\epsilon>0$, we have

$$
\begin{aligned}
& \left\|\mathcal{H}_{\Phi, 1}^{b_{0}} f_{1}\right\|_{L^{p}\left(\mathbb{R}^{n}\right)} \\
& \geq\left(\left.\left.\int_{|x|>\frac{1}{\epsilon}}\left|\int_{\{y|| y \mid \leq 1\} \cap S(x, y)} \frac{\Phi(y)}{|y|^{n}}\right| s(|y|)\right|^{-n / p-\epsilon} \log \frac{1}{|s(|y|)|} d y\right|^{p}|x|^{-n-p \epsilon} d x\right)^{\frac{1}{p}} \\
& \geq \omega_{n}^{\frac{1}{p}}(\epsilon p)^{-\frac{1}{p}} \epsilon^{\epsilon} \int_{\{y|| y \mid \leq 1\} \cap S(\epsilon, y)} \frac{\Phi(y)}{|y|^{n}}|s(|y|)|^{-n / p-\epsilon} \log \frac{1}{|s(|y|)|} d y \\
& =\epsilon^{\epsilon}\left\|f_{1}\right\|_{L^{p}\left(\mathbb{R}^{n}\right)} \int_{\{y|| y \mid \leq 1\} \cap S(\epsilon, y)} \frac{\Phi(y)}{|y|^{n}}|s(|y|)|^{-n / p-\epsilon} \log \frac{1}{|s(|y|)|} d y .
\end{aligned}
$$

It follows that

$$
\left\|\mathcal{H}_{\Phi, 1}^{b_{0}}\right\|_{L^{p}\left(\mathbb{R}^{n}\right) \rightarrow L^{p}\left(\mathbb{R}^{n}\right)} \geq \epsilon^{\epsilon} \int_{\{y|| y \mid \leq 1\} \cap S(\epsilon, y)} \frac{\Phi(y)}{|y|^{n}}|s(|y|)|^{-n / p-\epsilon} \log \frac{1}{|s(|y|)|} d y .
$$

Letting $\epsilon \rightarrow 0$ (using $\epsilon^{\epsilon} \rightarrow 1$ ), then we obtain

$$
\left\|\mathcal{H}_{\Phi, 1}^{b_{0}}\right\|_{L^{p}\left(\mathbb{R}^{n}\right) \rightarrow L^{p}\left(\mathbb{R}^{n}\right)} \geq \int_{|y| \leq 1} \frac{\Phi(y)}{|y|^{n}}|s(|y|)|^{-n / p} \log \frac{1}{|s(|y|)|} d y .
$$

So, combining the boundedness of $\mathcal{H}_{\Phi, 1}^{b}$, we obtain

$$
\int_{|y| \leq 1} \frac{\Phi(y)}{|y|^{n}}|s(|y|)|^{-n / p} \log \frac{1}{|s(|y|)|} d y<\infty .
$$

By a similar proof as that for Case 1, we get

$$
\int_{|y| \leq 1} \frac{\Phi(y)}{|y|^{n}}|s(|y|)|^{-n / p}<\infty .
$$


This completes the proof of part 1 ).

2) The proof for 2) is similar to that of 1$)$, except replacing the function $b_{0}(x)$ by $\log \frac{1}{|x|}$. We omit the details.

Acknowledgement. This work was supported partially by National Natural Science Foundation of China (Grant No. 11426204).

\section{REFERENCES}

1. J. Alvararez, M. Guzman-Partida and J. Lakey, Spaces of bounded $\lambda$-central mean oscillation, Morrey spaces, and $\lambda$-central Carleson measures, Collect Math. 51 (2000), 1-47.

2. K.F. Andersen, Boundedness of Hausdorff operators on $L^{p}\left(\mathbb{R}^{n}\right), H^{1}\left(\mathbb{R}^{n}\right)$ and BMO $\left(\mathbb{R}^{n}\right)$, Acta Sci. Math. (Szeged) 69 (2003), 409-418.

3. G. Brown and F. Móricz, Multivariate Hausdorff operators on the spaces $L^{p}\left(\mathbb{R}^{n}\right)$, J. Math. Anal. Appl. 271 (2002), no. 2, 443-454.

4. C. Carton-Lebrun and M. Fosset, Moyennes et quotients de Taylor dans BMO, Bull Soc. Roy. Sci. Liège 53 (1984), 85-87.

5. J.C. Chen, D.S. Fan and J. Li, Hausdorff operators on function spaces, Chinese Ann. Math., Ser. B 33 (2012), no. 4, 537-556.

6. J.C. Chen, D.S. Fan and C.J. Zhang, Multilinear Hausdorff operators and their best constants, Acta Math. Sin. (Engl. Ser.) 28 (2012), no. 8, 1521-1530.

7. J.C. Chen, D.S. Fan and C.J. Zhang, Boundedness of Hausdorff operators on some product Hardy type spaces, Appl. Math. J. Chinese Univ. Ser B, 27 (2012), no. 1, 114-126.

8. J.C. Chen and X.R. Zhu, Boundedness of multidimensional Hausdorff operators on $H^{1}\left(\mathbb{R}^{n}\right)$, J. Math. Anal. Appl. 409 (2014), 428-434.

9. N.M. Chuong and H.D. Huang, Bounds of weighted HardyCCesàro operators on weighted Lebesgue and BMO spaces, Integral Transforms Spec. Funct. 25 (2014), no.9, 697-710.

10. D.S. Fan and F.Y. Zhao, Multilinear Fractional Hausdorff Operators, Acta Math. Sin. Engl. Ser. 30 (2014), no. 8, 1407-1421.

11. Z.W. Fu, S.Z. Lu and W. Yuan, A weighted variant of Riemann-Liouville fractional integrals on $\mathbb{R}^{n}$, Abstr. Appl. Anal. (2012), Article ID 780132, 18 pages.

12. G.L. Gao, X.M. Wu and W.C. Guo, Some results for Hausdorff operators, Math. Inequal. Appl. 18 (2015), no. 1, 155-168.

13. C. Georgakis, The Hausdorff mean of a Fourier-Stieltjes transform, Proc. Amer. Math. Soc. 116 (1992), 465-471.

14. A. Hussain and G.L. Gao, Some new estimates for the commutators of n-dimensional Hausdorff operator, Appl. Math. J. Chinese Univ. Ser. B 29 (2014), no. 2, 139-150.

15. E. Liflyand and F. Móricz, The Hausdorff operator is bounded on the real Hardy space $H^{1}(\mathbb{R})$, Proc. Amer. Math. Soc. 128 (2000), 1391-1396.

16. A.G. Siskakis, Composition operators and the Ces'aro operator on $H^{p}$, J. London Math. Soc. 36 (1987), 153-164.

17. X.M. Wu and J.C. Chen, Best constants for Hausdorff operators on n-dimensional product spaces, Sci. China Ser. A 57 (2014), 569-578.

18. J. Xiao, $L^{p}$ and BMO bounds of weighted Hardy-Littlewood Averages, J. Math. Anal. Appl. 262 (2001), 660-666.

Xingzhi Colledge, Zhejiang Normal University, Jinhua 321004, China.

E-mail address: wuxm@zjnu.cn 